\title{
ПАССИВНЫЕ ОПТИЧЕСКИЕ WAN-сети
}

О.Карандин, магистрант МИРэА - Российский технологический университет / karandin.oleg@gmail.com, С.Портной, д.т.н., академик РАЕН, директор по развитию бизнеса компании "Комптек"

УДК 621.391.63, DOI: 10.22184/2070-8963.2019.82.5.44.46

Пассивная архитектура оптических WAN позволяет повышать пропускную способность транспортных сетей без увеличения затрат на инфраструктуру. В статье рассматриваются преимущества и недостатки этой архитектуры, изучается изменение подходов к проектированию сетей по сравнению с активной архитектурой.

\section{ВВеДЕНИЕ}

Объем передаваемого трафика в городских и региональных metro-сетях быстро растет. Это связано с повсеместным проникновением оптических сетей и ростом потребления пользователями медиаконтента. Кроме того, новое поколение мобильной связи, 5С, предъявляет высокие требования к пропускной способности и задержкам в транспортных backhaul-сетях, а также при использовании централизованной архитектуры C-RAN и в fronthaul-сетяx: от централизованного baseband-контроллера до радиомодулей на границе сети.

Операторы связи ищут способы увеличения пропускной способности metro-сетей при сохранении расходов на прежнем уровне. Одно из решений - минимизировать число дорогостоящих ROADM-мультиплексоров в узлах транспортной сети и допустить пассивное распространение сигналов. Это стало возможным благодаря достижениям в области электронной компенсации дисперсии, а также развитию настраиваемых оптических передатчиков и когерентных приемников.

Описанная архитектура была впервые предложена в [1] и называется FON (Filterless Optical Network). Экспериментальное внедрение таких сетей в Европе началось в 2012 году [2].

\section{КОНЦЕпЦИЯ}

Различия между традиционной архитектурой, основанной на активной коммутации с применением ROADM-мультиплексоров, и новой, filterless-архитектурой, изображены на рис.1 [3]. В сети с пассивной архитектурой сигналы в узлах не фильтруются и передаются дальше после достижения узла-приемника. Во избежание образования циклов и лазерного эффекта сеть делится на деревья, которые выделены на рисунке синим и красным пунктиром.

Активные оптические add-drop-мультиплексоры для разделения каналов с разными длинами волн используют дорогостоящие модули WSS (Wavelength selective switch, частотно-селективный коммутатор), реализованные или на MEMSтехнологии (Micro electro mechanical system, микроэлектромеханическая система) или как последовательность оптических интерферометров. Пассивные узлы FON-сети содержат лишь сплиттеры и сумматоры, а прием данных на требуемой длине волны осуществляется методом когерентной демодуляции, что значительно снижает САРЕХ. Кроме того, избавление от сложных электрооптических компонентов позволяет повысить надежность оборудования и уменьшить расходы на его обслуживание, а также снизить энергопотребление. Все это снижает расходы на OPEX. 


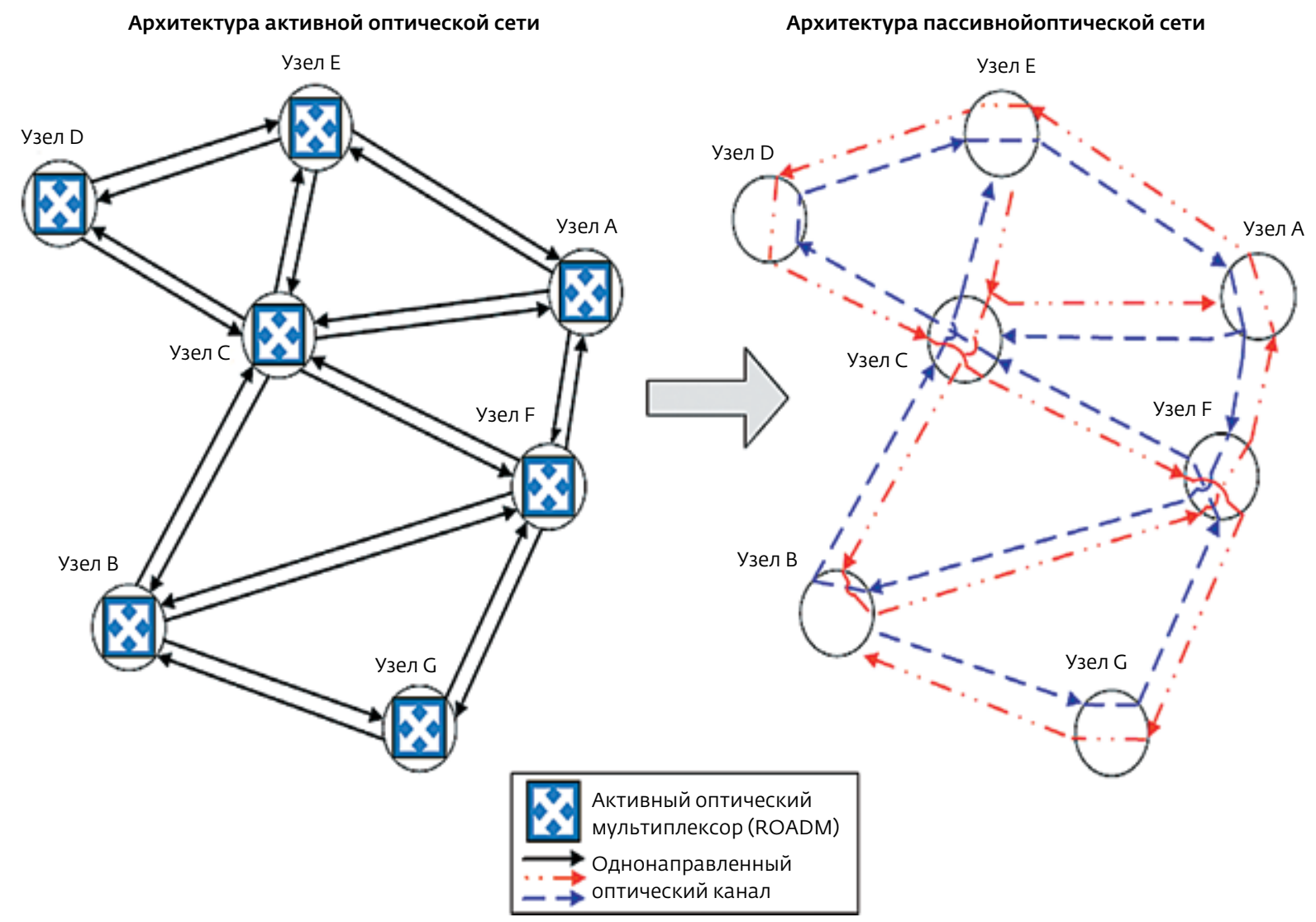

Рис.1. Традиционная активная и новая пассивная архитектура

Отсутствие WSS, жестко настроенных на определенную частотную сетку, позволяет реализовать динамическое распределение спектральных ресурсов. Объединяя частотные слоты по 12,5 ГГц, можно выделять каналы разной ширины, в зависимости от запросов пользователя.

Сигнал распространяется пассивно, поэтому не требуется дополнительных усилий для передачи multicast-трафика. Это особенно актуально в metro-сетях в связи с развитием систем кэширования контента на границе сети.

\section{РЕАЛИЗАЦИЯ И КОМПРОМИССЫ}

Новая архитектура требует разработки новых алгоритмов планирования сетей.

Так как сигналы распространяются беспрепятственно, сеть нужно разделить на отдельные деревья, чтобы не допустить образования циклов и лазерного эффекта. Из множества возможных разбиений топологии нужно выбрать одно, удовлетворяя требованиям связности и запрашиваемой емкости, а также физическим ограничениям на дальность распространения сигналов. Искать решение этой проблемы можно для задачи математического программирования, но пространство решений очень велико, поэтому предлагается использовать эвристические методы [4].

Проблема поиска резервных маршрутов частично решается за счет того, что каждый узел может входить в несколько деревьев, но в общем случае это является отдельной задачей [5]. Из-за отсутствия фильтрации использованную один раз длину волны нельзя использовать повторно внутри одного дерева. Необходимо распределить спектральные ресурсы между парами "передатчик - приемник" так, чтобы все они использовали разные длины волн. Эту задачу опять же можно решить оптимально, но в существующих 
исследованиях используются эвристические алгоритмы, например табу-поиск [4].

Сигнал, достигнув приемника, не фильтруется и продолжает распространяться по сети, поэтому часть емкости неизбежно теряется. Существует несколько подходов, позволяющих снизить потребление спектральных ресурсов. Во-первых, отказ от жесткой частотной сетки. В работе [6] показано, что это позволило сэкономить до 30\% частотных ресурсов. Во-вторых, на входных интерфейсах некоторых узлов могут быть установлены оптические фильтры, позволяющие повторно использовать некоторые длины волн в следующем за этим узлом сегменте сети [7]. В-третьих, активно развиваются оптические программируемые whitebох-устройства, в частности пассивные коммутаторы, позволяющие переконфигурировать способы соединения входных и выходных интерфейсов, напрямую или через сплиттеры и сумматоры. В рассмотренных в работе [8] случаях их применение позволило сократить использование спектральных ресурсов на $12 \%$.

Исследование [9] показывает, что, несмотря на неоптимальное использование спектра, применение данной архитектуры позволяет снизить стоимость построения городских и региональных сетей операторов, как транспортных, так и опорных.

Отсутствие фильтрации позволяет свободно распространяться не только сигналам, но и шумам, в частности шумам оптического усилителя (ASE). Это кардинальное отличие от актив ной архитектуры, в которой для определения отношения "сигнал - шум" (OSNR) нужно учитывать только шумы усилителей на пути от приемника к передатчику. В описываемой архитектуре на OSNR для данной пары приемника и передатчика может оказывать влияние шум от усилителей в других участках дерева, что требует разработки новой физической модели.

\section{ЗАКЛЮЧЕНИЕ}

Новая архитектура оптических metro-сетей, FON, стала возможна благодаря развитию электронных методов компенсации дисперсии и когерентного приема. Несмотря на неоптимальное использование спектральных ресурсов, она позволяет справляться с повышением нагрузки на сети операторов, снижая расходы на САРЕX и ОРЕХ.

Новая архитектура требует новых подходов к проектированию сетей, для формулировки которых необходимо проведение ряда исследований. Во-первых, требуется построение новой физической модели для оценки значений оптического отношения OSNR на приемнике. Во-вторых, необходимо построить эффективный алгоритм разбиения сети на деревья и распределения спектральных ресурсов. В-третьих, требуется построение алгоритма разбиения сети на деревья с учетом различных методов резервирования. Наконец, для дальнейшего снижения затрат на построение сети имеет смысл оптимизировать число используемых оптических усилителей.

\section{ЛИТЕРАТУРА}

1. Tremblay C. et al. Filterless Optical Networks: A Unique and Novel Passive WAN Network Solution. Optoelectron. Commun // Conf. OECC 2007. P. 12P-7. Yokohama, Japan, July 9-132007.

2. Gunkel $\mathbf{M}$. et al. Vendor-interoperable elastic optical interfaces: Standards, experiments and challenges // IEEE/OSA J. Opt. Commun. Netw. 2015. V. 7. No. 12. P. B184-B193.

3. Savoie G. et al. Physical layer validation of filterless optical networks // The 2010 European Conference on Optical Communication (ECOC 2010). P. P 5.08. Turin, Italy, 19-23 September 2010.

4. Archambault É., Xu Z., Tremblay C. et al. Routing and Spectrum Assignment in Elastic Filterless Optical Networks // IEEE/ACM Transactions On Networking. 2016. V. 24. No. 6.

5. Xu Z. et al. $1+1$ dedicated optical-layer protection strategy forfilterless optical networks // IEEE Commun. Lett. 2014. V. 18. No. 1. P. 98-101.

6. Xu Z., Tremblay C., Archambault É. et al. Flexible Bandwidth Assignment in Filterless Optical Networks // IEEE Commun. Lett. 2015. V. 19. No. 4. P. 565-568.

7. Khanmohamadi $\mathbf{S}$. et al. Semi-filterless optical network: a cost-efficient passive wide area network solution with effective resource utilization // 2011 Asia Communications and Photonics Conference and Exhibition (ACP). 2011.

8. Furdek $\mathbf{M}$. et al. Programmable Filterless Network Architecture Based on Optical White Boxes // 20th International Conference on Optical Network Design and Modeling (ONDM 2016), Cartagena, Spain. 9-12 May 2016.

9. Tremblay C. et al. Agile Optical Networking: Beyond Filtered Solutions // The 2018 Optical Networking and Communication Conference (OFC 2018). Paper M1A.5. 8-12 March 2018. 


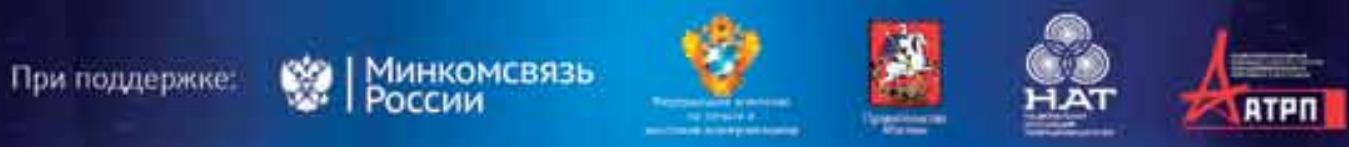
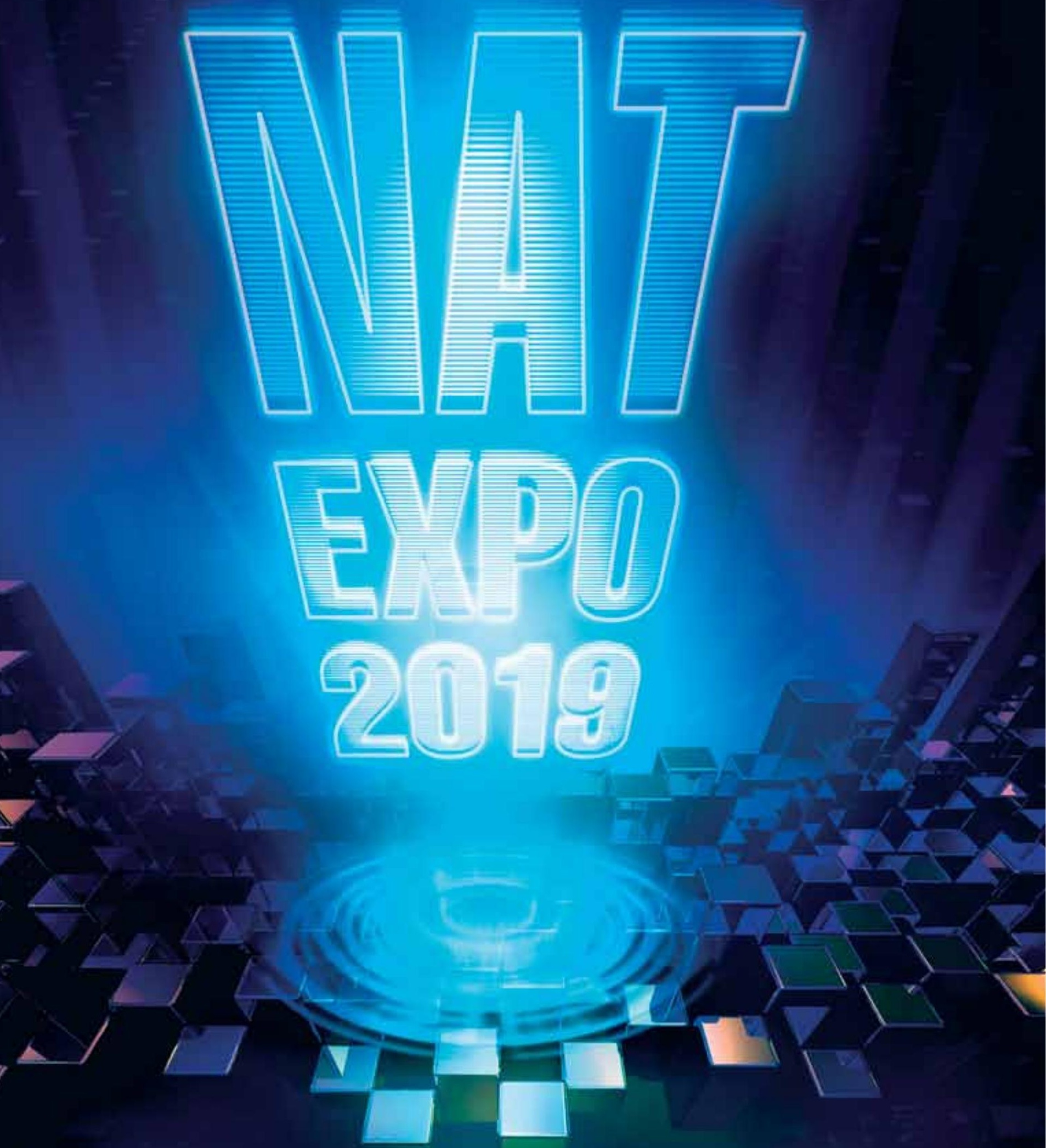

5-7 НОЯБРЯ, 2019

МОСКВА, ВДНХ

www.natexpo.ru

f. www.facebook.com/groups/NATEXPO 